\title{
MR-guided lumbar facet radiofrequency denervation for treatment of patients with chronic low back pain in an open 1.0 Tesla MRI system
}

\author{
Georg Böning ${ }^{1} \wedge$, Tony Hartwig ${ }^{2}$, Patrick Freyhardt ${ }^{3} \wedge$, Maximilian de Bucourt ${ }^{1} \wedge$, Ulf Teichgräber $^{4} \wedge$, \\ Florian Streitparth ${ }^{5 \wedge}$
}

${ }^{1}$ Department of Radiology, Charité-Universitätsmedizin Berlin, corporate member of Freie Universität Berlin, Humboldt-Universität zu Berlin, and Berlin Institute of Health, Berlin, Germany; ${ }^{2}$ Department of Musculoskeletal Surgery, Vivantes Hospital Spandau, Berlin, Germany; ${ }^{3}$ Faculty of Health, School of Medicine, University Witten/Herdecke, Witten, Germany; ${ }^{4}$ Department of Radiology, Friedrich-Schiller-University, Jena, Germany; ${ }^{5}$ Department of Radiology, Ludwig-Maximillians-University, München, Germany

Contributions: (I) Conception and design: F Streitparth, T Hartwig; (II) Administrative support: U Teichgräber; (III) Provision of study materials or patients: All authors; (IV) Collection and assembly of data: T Hartwig; (V) Data analysis and interpretation: F Streitparth, T Hartwig, G Böning; (VI) Manuscript writing: All authors; (VII) Final approval of manuscript: All authors.

Correspondence to: Prof. Dr. Florian Streitparth, MD, PhD. Department of Radiology, Ludwig-Maximillians-University, München, Germany. Email: florian.streitparth@med.uni-muenchen.de.

Background: To evaluate the feasibility, safety and efficacy of magnetic resonance imaging (MRI)-guided lumbar facet joint radiofrequency denervation (FRD) in patients with chronic low back pain.

Methods: The study consisted of two parts. First, a preclinical analysis using an ex vivo animal model was performed to define optimal technical parameters for ablation. Then, 17 patients with chronic lumbar facet joint pain syndrome were prospectively included and underwent MRI-guided FRD in an open 1.0-Tesla MRI. We analyzed technical feasibility and complications as well as clinical outcome in terms of subjective pain assessed on a numerical visual analogue scale (VAS) before and after 1 week/6 months after FRD. Clinical assessment was complemented by measurement of paravertebral muscle volume and fat content before the intervention and at 6-month follow-up.

Results: All interventions were technically successful without major complications. Initial VAS scores (median: 8, IQR: 1, range: 6-9, CI: 7.14-8.04) decreased significantly both after one week (median: 4, IQR: 5, range: 0-7, CI: 1.9-4.69, P=0.003) and after 6 months (median: 1, IQR: 6, range: 0-7, CI: 1.064.23, $\mathrm{P}<0.001$ ). Mean multifidus muscle volume increased significantly in the patient population (from $366.8 \pm 130.8 \mathrm{~cm}^{3}$ before to $435.4 \pm 146.7 \mathrm{~cm}^{3}$ after FRD, $\mathrm{P}=0.031$ ).

Conclusions: This proof of principle study shows MRI-guided FRD in an open 1.0-Tesla MRI system to be a potential therapy option for patients with chronic low back pain.

Keywords: Open MRI; MRI interventions; lumbar facet joint radiofrequency denervation; low back pain

Submitted Feb 08, 2021. Accepted for publication Apr 29, 2021.

doi: $10.21037 /$ atm-21-633

View this article at: https://dx.doi.org/10.21037/atm-21-633

\footnotetext{
^ ORCID: Georg Böning, 0000-0002-8819-6209; Patrick Freyhardt, 0000-0001-9705-8513; Maximilian de Bucourt, 0000-0002-6244-0496; Ulf Teichgräber, 0000-0002-4048-3938; Florian Streitparth, 0000-0003-1007-4057.
} 


\section{Introduction}

Chronic low back pain is one of the most commonly diagnosed conditions in developed countries with an enormous socioeconomic burden. In many patients, the condition is attributed to the facet joint pain syndrome with or without radiologically proven spondylarthrosis (1). For patients with a history of frustrating analgetic and physical therapy, corticoid/ analgesic infiltration of affected facet joints represents an adequate approach for supplementary diagnosis and first-line minimally invasive treatment (2). In patients who respond to infiltration, treatment can be extended to denervation of the facet joints to achieve a sustained therapeutic effect $(3,4)$. Facet joint radiofrequency denervation (FRD) using fluoroscopy or computed tomography (CT) for image guidance is an established method $(5,6)$.

However, there are several technical issues with fluoroscopy- and CT-guided FRD such as the radiation exposure for patient and interventionalist and poor soft tissue contrast. Higher radiation may be caused by difficult access to the nerve course of the medial dorsal ramus due to degeneratively enlarged facet portions, which can make denervation difficult in some cases. The lack of full threedimensional angulation of the CT image plane to monitor the position of the thermal applicator may limit the extent of medial dorsal ramus denervation and can thus result in insufficient facet denervation.

Successful magnetic resonance imaging (MRI)-guided interventions in the area of the spinal column have been reported for several indications, such as spinal injection and laser disc decompression (7-13). However, MRI has not yet been used for radiofrequency denervation of the facet joints. In this study, we therefore investigated the feasibility and clinical outcome of multiplanar MRI-guided lumbar FRD in an open 1.0 Tesla MRI scanner.

This article has been written in accordance with the MDAR reporting checklist (available at https://dx.doi. org/10.21037/atm-21-633).

\section{Methods}

\section{Study design/patient cohort}

This prospective, exploratory single-center study was conducted in accordance with the Declaration of Helsinki (as revised in 2013), approved by the institutional ethics commitee of our university hospital (EA1/071/09, EA1/301/12) and written informed consent was obtained from all patients.

Inclusion criteria: only patients with a preinterventional lumbar pain score of at least 6 on a visual analoge scale (VAS), spondylarthrosis detected by MRI ( $\geq$ Fujiwara $\left.2-3^{\circ}\right)$, and multiple positive facet joint infiltrations $(>75 \%$ pain relief) were included (14). At least 2 of the following 4 symptoms of facet joint pain syndrome were present: local pressure and knocking pain over one or more facets, worsening of low back pain with hyperextension and rotation of the lumbar spine, morning stiffness in the lumbar spine or increased pain in the morning with pseudoradicular radiation into the buttocks or thigh. Exclusion criteria were: progressive neurological deficits due to neuroforaminal and spinal canal stenosis, evidence of lumbosacral radiculopathy, spinal and zygapophyseal cysts, spondylolisthesis, pre- or postinterventional surgery of the lumbar spine, BMI $>40 \mathrm{~kg} / \mathrm{m}^{2}$, skin infection, circulatory disorders, allergic reactions to local anesthesia, and relevant neurological, cardiological or malignant disease. Indications for facet joint denervation were jointly established by orthopedic surgeons and interventional radiologists.

\section{MRI system and MR-compatible interventional equipment}

All interventions were performed in an open 1.0-Tesla MRI system with vertical orientation of the main magnetic field (Panorama HFO, Philips, Best, Netherlands). The open configuration allows almost $360^{\circ}$ patient access in the magnetic isocenter and thus provides good conditions for MR-imageguided interventions. The equipment used in the open MRI system included a workstation with complete scanner control, an MRI-compatible in-room monitor, and an MRI-compatible wireless PC mouse for starting interventional acquisitions and switching imaging planes. A flexible solenoid superficial coil (Multipurpose L, Philips, Best, Netherlands) with a diameter of $21 \mathrm{~cm}$ was used for all procedures.

All facet denervations were performed using a bipolar radiofrequency (RF) system (CelonLab POWER, Celon AG medical instruments, Teltow, Germany) with a maximum output power of $250 \mathrm{~W}$ and a frequency of $470 \mathrm{kHz}$. The MRI-compatible RF applicator (CelonProSurge MR 150-T20, Celon AG medical instruments, Teltow, Germany) has an electrode length of $20 \mathrm{~mm}$, a shaft length of $150 \mathrm{~mm}$, and a diameter of 15 gauge $(1.79 \mathrm{~mm})$.

\section{Preclinical analysis}

As no presets for use of the MR-compatible RF applicator for facet joint denervation were avaible at the time of the study, we first carried out ablation tests in an ex vivo porcine 

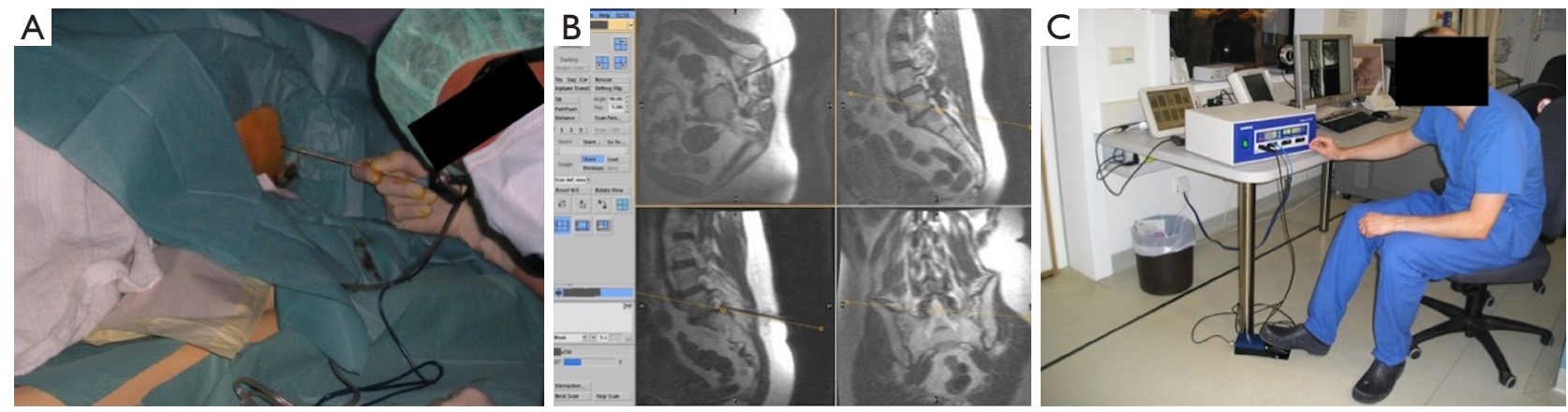

Figure 1 Setting and planning of MRI-guided FRD. (A) Interventionalist with the RF applicator in hand adjacent to skin entry point; (B) interface for MR guidance with interactive viewports in different planes (axial, sagittal and coronal); positioning of the applicator matches preinterventional planning, (C) operation of RFA power-supply from outside the interventional suite with connected cables passing through a wall hole. MRI, magnetic resonance imaging; FRD, facet joint radiofrequency denervation; RF, radiofrequency; RFA, radiofrequency ablation.

model to identify the optimal power, energy, and application duration to be selected in relation to the size of the ablation area to be achieved. For this purpose, radiofrequency ablations at $5 \mathrm{~W}$ and different target energy inputs of $100-200 \mathrm{~J}$ (in $20 \mathrm{~J}$ steps) were combined with different application durations, and the ablation areas/shapes achieved in the animal muscle were subjectively evaluated by two experienced interventionalists in consensus. The aim was to determine the optimal energy input regarding area/shape in terms of denervation effectiveness and maximum protection of surrounding tissue such as paravertebral muscles.

\section{Clinical application}

Denervation treatment was performed on an outpatient basis. The interventions were uneventful, and all patients left the hospital after circulatory monitoring for one hour after the procedure.

The interventional team included an MRI technician for coil positioning and pre- and postinterventional imaging, a nurse for sterile patient and equipment preparation, an interventional radiologist, and an orthopedic surgeon. Patients were positioned in $90^{\circ}$ lateral position, and the lumbar interventional area was covered sterilely in the usual manner (Figure 1). In order to achieve optimal image quality, the radiofrequency coil was positioned as close as possible to the lumbar puncture site in a position orthogonal to the main magnetic field (B0).

The MRI sequence protocol is presented in Table 1. Multislice T1- and T2-weighted (w) fast spin echo (FSE) sequences were used for preprocedural planning and localization of the target anatomy (Figure 2). An interactive proton density (PD)-w FSE sequence was used to determine the exact skin entry point using the finger-point technique and to direct the applicator needle toward the target anatomy in near real-time (acquisition time: $2 \mathrm{~s}$ ) (Figure 2). A local anesthetic $\left(2 \mathrm{~mL}\right.$ prilocaine $1 \%$, Xylonest ${ }^{\mathrm{TM}}$ $1 \%$, AstraZeneca, Wedel, Germany) was administered subcutaneously in the area of the predetermined puncture sites and around the facet joints to be treated.

After a small skin incision, the RF applicator was positioned parallel to the medial branch of the respective dorsal ramus in approx. $15^{\circ}$ inferosuperior puncture direction with posterolateral access. Here, the medial dorsal ramus is located in a groove along the medial-posterior surface of the transverse process (15). For upward advancement of the $\mathrm{RF}$ applicator, a puncture site approximately one level below the target site was used. First, the needle should be used to establish bone contact with the transition of the lateral surface of the superior articular process and the transverse process. After this step, the probe was brought into the final position-parallel to the nerve.

For the RFA procedure, a $2 \mathrm{~W}$ test application was carried out for approx. 2-3 s to rule out irritation of the nerve root after applicator positioning. Each facet joint was treated until the defined autostop settings of the RF generator were reached, and a strongly T2-w fat-saturated SPIR sequence was acquired for assessment of postinterventional effects.

\section{Technical feasibility and safety}

Image quality of real-time MRI was evaluated subjectively 
Table 1 Imaging protocols in open 1.0-T MRI system

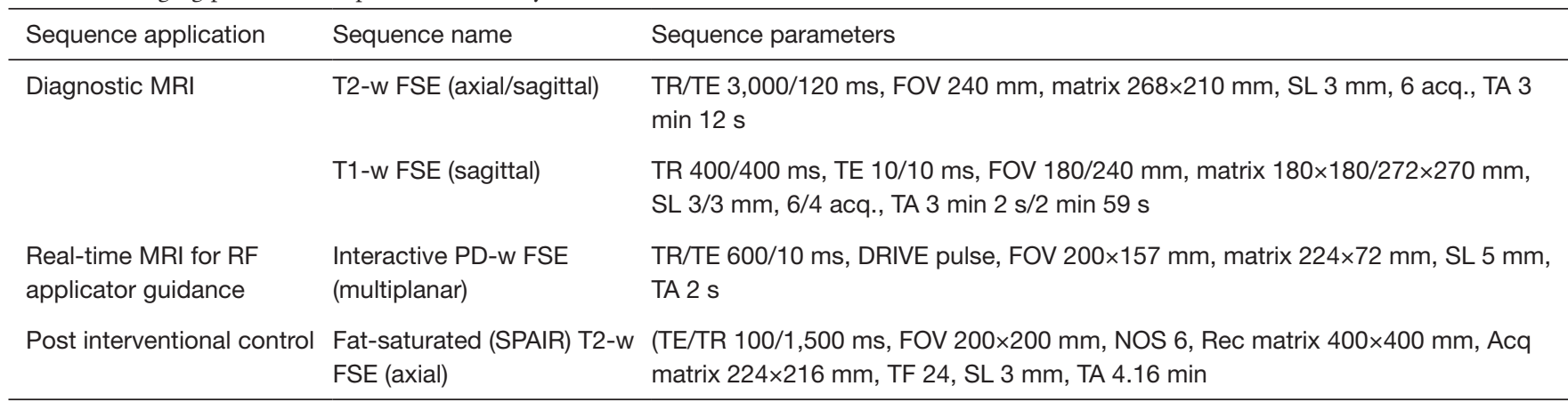

DRIVE, driven equilibrium RF reset pulse; FOV, field of view; FSE, fast spin echo; MRI, magnetic resonance imaging; NSA, number of signal averages; PDw FSE, proton density weighted fast spin echo; SL, slice thickness; SPAIR, spectral attenuated inversion recovery; SPIR; spectral presaturation with inversion recovery; TA, acquisition time; TE, echo time; TR, repitition time. Table reprint from Streitparth et al. with kind permission from Springer Nature (11).

regarding accurate and safe RF applicator guidance by two experienced interventional radiologists in consensus. The procedure was defined as technically successful when the applicator reached the corresponding target position at the level of the respective medial dorsal ramus branch with MR fluoroscopy for guidance, and FRD was accomplished. Complications were recorded according to the Society of Interventional Radiology (SIR) clinical practice guidelines (16).

\section{Clinical outcome}

Back pain was evaluated clinically using follow-up questionnaires with a VAS for subjective assessment of low back pain ranging from $0(0=$ no pain $)$ to 10 $(10=$ maximum pain intensity). VAS scores were obtained before the intervention and at follow-up after one week and six months.

\section{Volumetric assessment}

To complement the results of subjective pain assessment, we measured paravertebral muscle volumes and fat content. Diagnostic MRI examinations using the same protocols were performed for intervention planning and for follow-up at 6-month intervals. All volumes were measured manually by an experienced reader, and results over time were compared.

The volumetric measurements were performed as described before by Hartwig et al. using Amira v.5.2.0 (Visage Imaging, San Diego, CA, USA) (17). The craniocaudal extent of volumetric measurement was defined according to the treated segments following the definition of Hartwig et al. were the middles of intervertebral discs in the sagittal plane were used to define the superior and inferior margins of the region of interest (17). For example, if segment L4/5 was treated, the region of interest was defined from the center of the L3/4 disc to the center of the L5/S1 disc. The measurements were than performed in the axial $\mathrm{T} 2 \mathrm{w}$ with $3 \mathrm{~mm}$ slice thickness.

\section{Statistical analysis}

Graphics were created with GraphPad Prism v. $5^{\oplus}$ (GraphPad Software, San Diego, CA, USA). Statistical analysis was performed with IBM SPSS Statistics 26 for Windows 10 (IBM Corp., Armonk, NY, USA). Descriptive evaluation of the data was done using common measures of location (median, mean), dispersion measures [standard deviation (SD), range from minimum ( $\mathrm{min}$ ) to maximum (max), interquartile range (IQR)], and frequency tables. For exploratory data analysis, we used the Wilcoxon signedrank test (interval scaled data) and Friedman`s two-way analysis of variance by ranks (ordinal scaled data). Statistical significance was assumed for $\mathrm{P}<0.05$, and Bonferroni correction was used.

\section{Results}

\section{Patient population}

In this study, 17 patients ( 7 women/10 men; median age: 58 years, range: $37-83$ years) with chronic lumbar facet joint pain syndrome underwent MRI-guided facet joint radiofrequency denervation. Due to the dual nerve supply of 

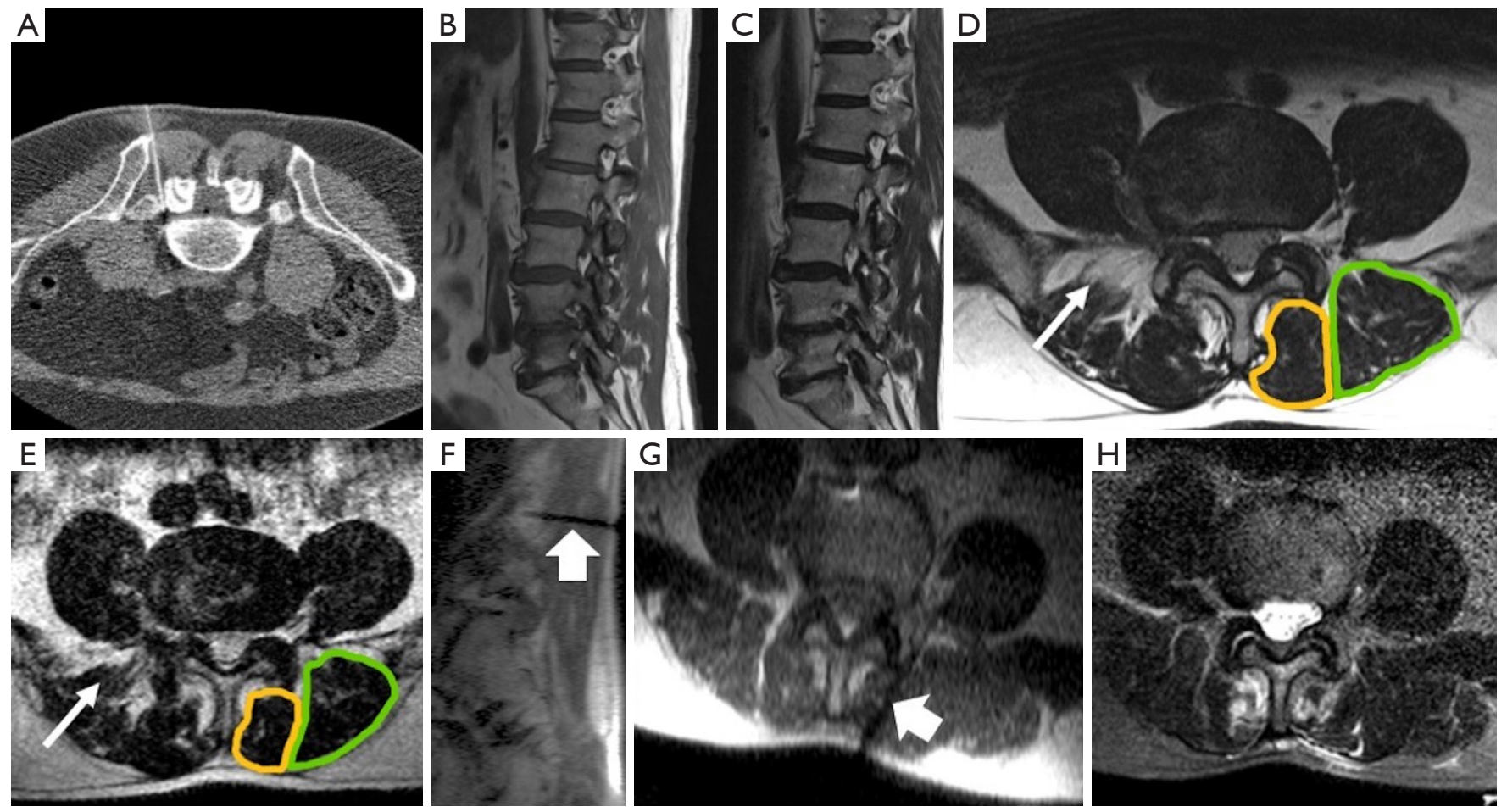

Figure 2 Clinical case. 68-year-old male patient with therapy-refractory low back pain, VAS score reduction from 8 to 5 (1 week after intervention and in mid-term after 6 months); (A) the patient had a history of several positive CT-guided facet joint infiltrations as a prerequisite for the indication for MRI-guided FRD; (B) T1w parasagittal TSE image before intervention; (C) T2w parasagittal TSE image before intervention; (D) T2w axial TSE image before intervention: arrow indicates fatty degeneration of the muscle, also note spondylarthrosis at L4/5; (E) T2w axial TSE image acuqired 6 months after intervention: arrow: recovery of muscle tissue; (F) real-time sagittal PDw FSE image during intervention: arrowhead indicates RF applicator; $(\mathrm{G})$ real-time PDw axial FSE image acquired during intervention: arrowhead indicates RF applicator in final position adjacent to the symptomatic facet joint; (H) T2w SPAIR image obtained after denervation showing local anesthetic deposition in the access route and no signal alterations in the area of vulnerable tissues such as nerve root. Illustration of ROI positioning for measuring muscle volumes: green: longissimus muscle, yellow: multifidus muscle. CT, computed tomography; FRD, facet joint radiofrequency denervation; MRI, magnetic resonance imaging; PDw FSE, proton density weighted fast spin echo; RF, radiofrequency; ROI, region of interest; SPAIR, spectral attenuated inversion recovery; TSE, turbo spin echo; VAS, visual analogue scale.

each individual segment by descending fibers, the adjacent cranial segment was always treated as well. Segments L2/3 $(\mathrm{n}=1), \mathrm{L} 3 / 4(\mathrm{n}=5)$ and L4/5 (11) were primary targets. In 11 patients, two segments ( $\mathrm{n}=66$ ablations) and in four patients, three segments ( $n=32$ ablations) were denervated in the same session, resulting in a total of 106 facet joint ablations. Further patient details are summarized in Table 2.

\section{Technical feasibility and safety}

Optimal ablation settings identified experimentally were: $5 \mathrm{~W}, 100 \mathrm{~J}$, and $20 \mathrm{~s}$ application duration, resulting in an oval ablation area of $4 \mathrm{~mm} \times 7 \mathrm{~mm}$.
All procedures were technically successful. No major complications occurred during or after the interventions. Mild injection site pain was observed in all patients.

Image quality of the interactive PD-w FSE sequence was adequate, allowing identification of the targeted anatomical landmarks and confirmation of correct needle positioning parallel to the medial dorsal ramus in all patients.

\section{Clinical outcome}

Subjective pain scores were improved after the intervention. Preinterventional initial scores (median: 8 , IQR: 1 , range: 6-9, CI: 7.14-8.04) decreased significantly both after one 
Table 2 Details of patient population

\begin{tabular}{|c|c|c|c|c|}
\hline & $\mathrm{n}$ & $\%$ of total & Median/IQR & $95 \% \mathrm{Cl}$ \\
\hline \multicolumn{5}{|c|}{ Site of primary target segment } \\
\hline L2/3 & 1 & 5.9 & & \\
\hline L3/4 & 5 & 29.4 & & \\
\hline $\mathrm{L} 4 / 5$ & 11 & 64.7 & & \\
\hline \multicolumn{5}{|c|}{ High-intensity zone } \\
\hline Yes & 5 & 29.4 & & \\
\hline No & 12 & 70.6 & & \\
\hline \multicolumn{3}{|c|}{ Grade of disc degeneration [Pfirmann] } & $3 / 1$ & $2.90-3.69$ \\
\hline \multicolumn{3}{|c|}{ Endplate changes [Modic] } & $1 / 2$ & $0.44-1.44$ \\
\hline \multicolumn{3}{|c|}{ Grade of spondylarthosis [Fujiwara] } & $3 / 1$ & $2.46-2.95$ \\
\hline
\end{tabular}

Study population of 17 patients (7/10 female/male; median age: 58 years, range $37-83$ years) with chronic lumbar facet joint pain syndrome who underwent MRI-guided facet joint radiofrequency denervation. $\mathrm{Cl}$, confidence interval; IQR, interquartile range.

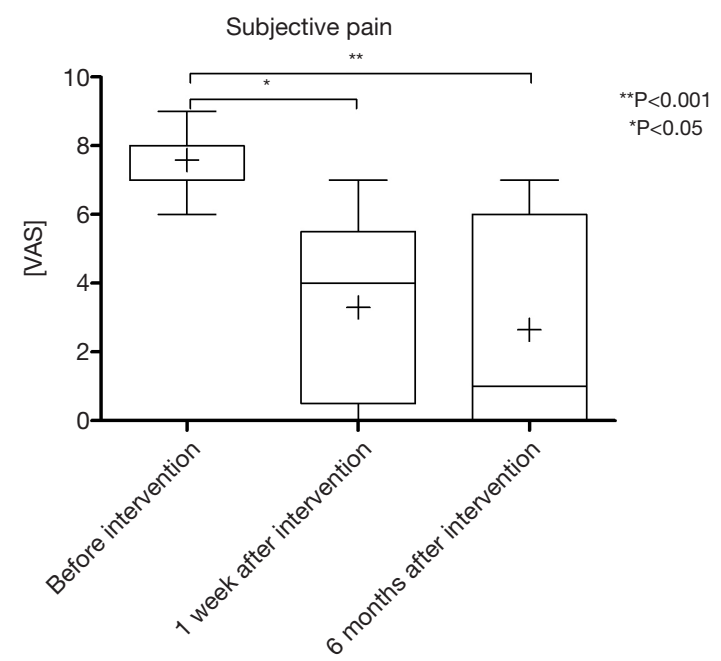

Figure 3 Subjective pain. Subjective pain scores (VAS) improved after the intervention in our patient population. Initial scores decreased significantly both after one week and after six months.

week (median: 4, IQR: 5, range: 0-7, CI: 1.9-4.69, $\mathrm{P}=0.003$ ) and after 6 months (median: 1, IQR: 6 , range: $0-7$, CI: $1.06-4.23, \mathrm{P}<0.001$ ) (Figure 3).

\section{Volume measurement}

Mean multifidus muscle volume before/after the

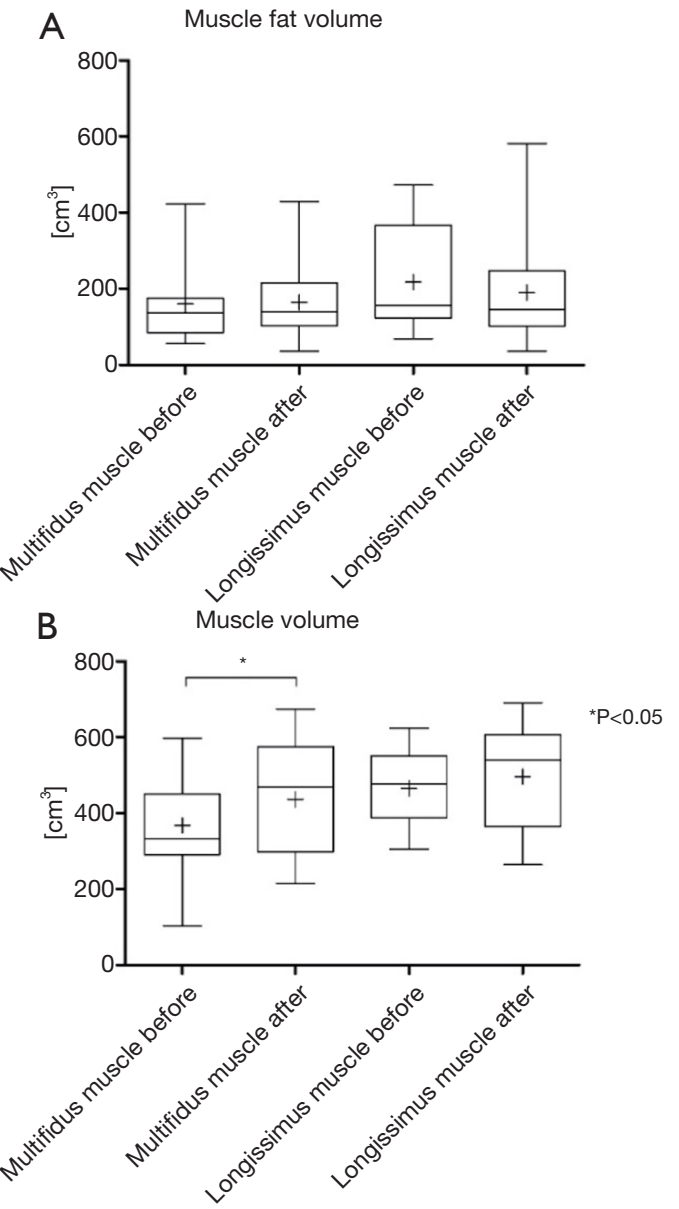

Figure 4 Muscle volumes. Mean volume of multifidus muscle before and after the intervention differed significantly. No significant differences were observed for the other volumes analyzed.

intervention increased significantly in our patient population $\left(366.8 \pm 130.8 \mathrm{~cm}^{3} / 435.4 \pm 146.7 \mathrm{~cm}^{3}, \mathrm{P}=0.031\right)$. No significant differences were observed for the other volumes analyzed (Figure 4).

\section{Discussion}

This proof-of-principle study describes the feasibility of real-time MRI-guided bipolar radiofrequency denervation of lumbar facet joints in patients with chronic lumbar pain syndrome in an open 1.0-Tesla MRI system. Facet joint pain is thought to be mediated by the neural supply of the facet joint capsule. The joints have a rich innervation arising from the medial and lateral branches of the dorsal rami with dual innervation from the medial branches arising from the 
posterior rami (18). Studies have shown that image-guided denervation of the facets is a safe and effective therapeutic option when indicated and can prevent or postpone surgical treatment (2). In addition to alcohol ablation, radiofrequency denervation using $\mathrm{X}$-ray fluoroscopy or CT guidance has become an established treatment option $(3,4,19)$. Significant pain reduction after denervation for chronic lumbar pain syndrome was reported for a 6-month interval (19) and is confirmed by the results of our study.

Previous studies suggest that MRI-assisted facet infiltration is safe and effective (7-9). The lack of ionizing radiation is advantageous for both patients and interventionalists. Furthermore, MRI navigation has additional advantages. For example, the better soft tissue contrast and especially the multiplanar imaging capabilities of MRI make it easier to position the RF applicator compared to fluoroscopy and CT. MR imaging allows $15^{\circ}$ inferosuperior angling of the RF applicator with complete visualization of the applicator. This allows a higher contact length to the medical dorsal ramus for optimized denervation.

We achieved technically successful MR-guided RF denervation of the facet joints in all patients without major complications. The real-time PD-w FSE sequence enabled precise multiplanar applicator positioning with good visualization of spinal structures and optimal artifact properties for applicator imaging. This was accomplished by a complex interaction of material and alignment of the applicator to the main magnetic field (B0), the sequence type, and parameters applied for the vertically oriented magnetic field strength of 1.0 Tesla (7). In comparison to CT, MRI depicts spinal anatomy in greater detail and facilitates applicator positioning due to muliplanar navigation with unlimited selection of image planes (20). In our study, interventions were performed with patients in lateral position, which allows the best possible coil positioning and hence optimal signal amplification (7). In addition, an MRI quadrupole imaging coil (butterfly) was developed for spinal interventions in a vertical 1.0-T MRI system and the use of this surface coil may allow spinal interventions to be performed in a prone position comparable to CT (21). Prone positioning ensures access to a larger area for spinal interventions and might be more familiar to interventionalists, especially to those with little to moderate experience. The durations of interventions were not recorded in this study. The interventionalist performing the procedures estimated the time to be comparable to that of fluoroscopyor CT-guided denervation after an initial learning curve, due to real-time MR fluoroscopy navigation. Furthermore, the development of dedicated sequences can improve the benefit and safety of MRI-guided interventions (22). Realtime thermometry was reported to technically optimize MRI-guided thermoablation (11,12,23-25). Other technical advances may enable new applications in the future $(26,27)$.

The use of a bipolar RF applicator contributed to the safety of the interventions performed in this study (28). Known risks such as current flow across a patient-neutral electrode (monopolar systems) are prevented by the use of a coaxial electrode (29). Current flow in a bipolar system solely occurs in the distal portion of the applicator. The fact that very low power is required for effective denervation further reduces the risk of unwanted thermal damage. Finally, the autostop function of the applicator which we defined in a preclinical experiment prevents damage to adjacent subcutaneous and muscle tissue as well as to structures at risk such as the nerve root.

A disadvantage of MRI-guided interventions is the currently still limited availability of open MRI scanners. However, techniques for performing interventional MRI guidance in closed-bore systems have been reported. In short wide-bore systems, needles can be advanced with the extended arm using real-time imaging. In standard magnets, control and workflow may be improved by remote operation using robotic or manual driving elements (30).

Regarding clinical outcome, we observed significant pain relief (median VAS scores of 8 to 1), which correlated with an increase in muscle volume (multifidus muscle) after 6 months. The underlying mechanism for this observation is that pain can lead to a sparing of the corresponding segments with subsequent hypotrophy of muscles and increasing fat content (31). Consequently, successful pain therapy is expected to reverse hypotrophy (32). This underlines the benefit of MRI-guided FRD with prompt pain relief (median VAS scores of 8 to 4 one week after denervation), allowing the patient to undergo optimized physiotherapy aimed at improving spinal muscle volume and ensuring sustained pain control.

Besides ethanol and radiofrequency denervation, there are other treatment options such as MRI-guided focused ultrasound ablation or cryoneurolysis that have shown promising results in animals and humans $(33,34)$. Studies comparing different procedures would be desirable and should be performed in the future.

Our study has some limitations. First, we used subjective pain scores without further verification. In addition, patients were asked to report the response to treatment by telephone (not blinded), which could have 
affected outcome results. Second, a follow-up period of 6 months is relatively short, as pain is bound to recur due to nerve regeneration, and a longer follow-up period would be helpful to analyze the duration of the therapeutic effect and the time when patients may need a repeat procedure. Third, as this was a preliminary study, we did not include a control group to compare MRI guidance with fluoroscopy or CT. Fourth a prospective case number planning was not performed because valid data on the expected effect size are available for CT-guided RFA, but it was not possible to predict with certainty whether comparable effects could be achieved with the MRI-guided procedure. However, the rough estimation of the effect size for this procedure, which is possible from the data of this pilot study, can be used to prospectively generate a sufficient number of cases at adequate test power in following studies. Fifth, this study was performed in an open MRI system, which is not the most common type available. Nevertheless, our experience is transferable to state-of-the-art wide- and short-bore tunnel systems, which are most appropriate for performing MR interventions. For this transfer, some requirements should be considered, such as modifications in patient and coil positioning and possible parameter modifications of the interactive MR sequence. Future studies in larger patient populations should investigate the clinical significance of the achieved pain reduction and long-term efficacy.

In conclusion, this proof-of-principle study shows that MRI-guided facet joint radiofrequency denervation (FRD) in an open 1.0-Tesla MRI system is a promising treatment option for patients with chronic lumbar pain syndrome.

\section{Acknowledgments}

The authors thank Bettina Herwig for professional English editing.

Funding: None.

\section{Footnote}

Reporting Checklist: The authors have completed the MDAR reporting checklist. Available at https://dx.doi. org/10.21037/atm-21-633

Data Sharing Statement: Available at https://dx.doi. org/10.21037/atm-21-633

Conflicts of Interest: All authors have completed the ICMJE uniform disclosure form (available at https://dx.doi. org/10.21037/atm-21-633). The authors have no conflicts of interest to declare.

Ethical Statement: The authors are accountable for all aspects of the work in ensuring that questions related to the accuracy or integrity of any part of the work are appropriately investigated and resolved. The study was conducted in accordance with the Declaration of Helsinki (as revised in 2013). The study was approved by the institutional ethics commitee of Charite Universitätsmedizin Berlin (EA1/071/09, EA1/301/12), and informed consent was obtained from all study participants.

Open Access Statement: This is an Open Access article distributed in accordance with the Creative Commons Attribution-NonCommercial-NoDerivs 4.0 International License (CC BY-NC-ND 4.0), which permits the noncommercial replication and distribution of the article with the strict proviso that no changes or edits are made and the original work is properly cited (including links to both the formal publication through the relevant DOI and the license). See: https://creativecommons.org/licenses/by-nc-nd/4.0/.

\section{References}

1. Urits I, Burshtein A, Sharma M, et al. Low Back Pain, a Comprehensive Review: Pathophysiology, Diagnosis, and Treatment. Curr Pain Headache Rep 2019;23:23.

2. Sehgal N, Shah RV, McKenzie-Brown AM, et al. Diagnostic utility of facet (zygapophysial) joint injections in chronic spinal pain: a systematic review of evidence. Pain Physician 2005;8:211-24.

3. Leggett LE, Soril LJ, Lorenzetti DL, et al. Radiofrequency ablation for chronic low back pain: a systematic review of randomized controlled trials. Pain Res Manag 2014;19:e146-53.

4. Joo YC, Park JY, Kim KH. Comparison of alcohol ablation with repeated thermal radiofrequency ablation in medial branch neurotomy for the treatment of recurrent thoracolumbar facet joint pain. J Anesth 2013;27:390-5.

5. Juch JNS, Maas ET, Ostelo R, et al. Effect of Radiofrequency Denervation on Pain Intensity Among Patients With Chronic Low Back Pain: The Mint Randomized Clinical Trials. JAMA 2017;318:68-81.

6. Lee CH, Chung CK, Kim CH. The efficacy of conventional radiofrequency denervation in patients with chronic low back pain originating from the facet joints: a meta-analysis of randomized controlled trials. Spine J 
2017;17:1770-80.

7. Streitparth F, Walter T, Wonneberger U, et al. Imageguided spinal injection procedures in open high-field MRI with vertical field orientation: feasibility and technical features. Eur Radiol 2010;20:395-403.

8. Streitparth F, De Bucourt M, Hartwig T, et al. Real-time MR-guided lumbosacral periradicular injection therapy using an open 1.0-T MRI system: an outcome study. Invest Radiol 2013;48:471-6.

9. Freyhardt P, Hartwig T, De Bucourt M, et al. MR-guided facet joint injection therapy using an open 1.0-T MRI system: an outcome study. Eur Radiol 2013;23:3296-303.

10. Streitparth F, Althoff C, Jonczyk M, et al. Tailored interactive sequences for continuous MR-image-guided freehand biopsies of different organs in an open system at 1.0 tesla (T) - Initial experience. Biomed Tech (Berl) 2017;62:557-63.

11. Streitparth F, Hartwig T, Walter T, et al. MR guidance and thermometry of percutaneous laser disc decompression in open MRI: an initial clinical investigation. Eur Radiol 2013;23:2739-46.

12. Streitparth F, Walter T, Wonneberger U, et al. MR guidance and thermometry of percutaneous laser disc decompression in open MRI: an ex vivo study. Cardiovasc Intervent Radiol 2014;37:777-83.

13. Namba H, Kawasaki M, Izumi M, et al. Effects of MRgFUS Treatment on Musculoskeletal Pain: Comparison between Bone Metastasis and Chronic Knee/Lumbar Osteoarthritis. Pain Res Manag 2019;2019:4867904.

14. Fujiwara A, Tamai K, Yamato M, et al. The relationship between facet joint osteoarthritis and disc degeneration of the lumbar spine: an MRI study. Eur Spine J 1999;8:396401.

15. Lau P, Mercer S, Govind J, et al. The surgical anatomy of lumbar medial branch neurotomy (facet denervation). Pain Med 2004;5:289-98.

16. Sacks D, McClenny TE, Cardella JF, et al. Society of Interventional Radiology clinical practice guidelines. J Vasc Interv Radiol 2003;14:S199-202.

17. Hartwig T, Streitparth F, Gross C, et al. Digital 3-dimensional analysis of the paravertebral lumbar muscles after circumferential single-level fusion. J Spinal Disord Tech 2011;24:451-4.

18. Bogduk N. Clinical anatomy of the lumbar spine and sacrum. 4th ed. New York: Churchhill Livingstone; 2005.

19. McCormick ZL, Marshall B, Walker J, et al. LongTerm Function, Pain and Medication Use Outcomes of
Radiofrequency Ablation for Lumbar Facet Syndrome. Int J Anesth Anesth 2015;2:028.

20. Stojanovic MP, Sethee J, Mohiuddin M, et al. MRI analysis of the lumbar spine: can it predict response to diagnostic and therapeutic facet procedures? Clin J Pain 2010;26:1105 .

21. Rump JC, Streitparth F, Boning G, et al. Evaluation of a MR-quadrupole imaging coil for spinal interventions in a vertical 1.0 T MRI. Magn Reson Med 2012;68:600-5.

22. Makowski MR, Jonczyk M, Streitparth F, et al. Interactive near-real-time high-resolution imaging for MR-guided lumbar interventions using ZOOM imaging in an open 1.0 Tesla MRI system--initial experience. Biomed Tech (Berl) 2015;60:533-9.

23. Bazrafshan B, Koujan A, Hubner F, et al. A thermometry software tool for monitoring laser-induced interstitial thermotherapy. Biomed Tech (Berl) 2019;64:449-57.

24. Wonneberger U, Schnackenburg B, Wlodarczyk W, et al. Evaluation of thermometric monitoring for intradiscal laser ablation in an open $1.0 \mathrm{~T}$ MR scanner. Int J Hyperthermia 2010;26:295-304.

25. Wonneberger U, Schnackenburg B, Wlodarczyk W, et al. Intradiscal temperature monitoring using double gradientecho pulse sequences at 1.0T. J Magn Reson Imaging 2010;31:1499-503.

26. Hensen B, Kagebein U, Gutberlet M, et al. Wireless video transmission into the MRI magnet room: implementation and evaluation at $1.5 \mathrm{~T}, 3 \mathrm{~T}$ and 7T. Biomed Tech (Berl) 2019;64:373-82.

27. Schmidt M, Krug JW, Rosenheimer MN, et al. Filtering of ECG signals distorted by magnetic field gradients during MRI using non-linear filters and higher-order statistics. Biomed Tech (Berl) 2018;63:395-406.

28. Bruners P, Lipka J, Gunther RW, et al. Bipolar radiofrequency ablation: is the shape of the coagulation volume different in comparison to monopolar RF-ablation using variable active tip lengths? Minim Invasive Ther Allied Technol 2008;17:267-74.

29. Burton CV. Percutaneous radiofrequency facet denervation. Appl Neurophysiol 1976-1977;39:80-6.

30. Busse H, Kahn T, Moche M. Techniques for Interventional MRI Guidance in Closed-Bore Systems. Top Magn Reson Imaging 2018;27:9-18.

31. Goubert D, Oosterwijck JV, Meeus M, et al. Structural Changes of Lumbar Muscles in Non-specific Low Back Pain: A Systematic Review. Pain Physician 2016;19:E985-E1000.

32. Bierry G, Kremer S, Kellner F, et al. Disorders of 
paravertebral lumbar muscles: from pathology to crosssectional imaging. Skeletal Radiol 2008;37:967-77.

33. Weeks EM, Platt MW, Gedroyc W. MRI-guided focused ultrasound (MRgFUS) to treat facet joint osteoarthritis low back pain--case series of an innovative new technique.
Eur Radiol 2012;22:2822-35.

34. Kaye EA, Monette S, Srimathveeravalli G, et al. MRIguided focused ultrasound ablation of lumbar medial branch nerve: Feasibility and safety study in a swine model. Int J Hyperthermia 2016;32:786-94.

Cite this article as: Böning $G$, Hartwig T, Freyhardt P, de Bucourt M, Teichgräber U, Streitparth F. MR-guided lumbar facet radiofrequency denervation for treatment of patients with chronic low back pain in an open 1.0 Tesla MRI system. Ann Transl Med 2021;9(13):1056. doi: 10.21037/atm-21-633 